\title{
Determinates That Influence Food Comsumption among Older Members of a Midwest Community
}

\author{
Deanna Pucciarelli, Adrienne Thomas \\ Department of Family and Consumer Sciences, Ball State University, Muncie, USA. \\ Email: dpucciarelli@bsu.edu
}

Received June 27 $7^{\text {th }}, 2011$; revised September $19^{\text {th }}, 2011$; accepted September $26^{\text {th }}, 2011$.

\begin{abstract}
The goal of this study was to record Muncie, Indiana residents' change in eating habits over time. Objectives: 1) Identify key determinants that influence a change in participants' eating habits; 2) Analyze the data for convergent themes among participants and draw patterns; and 3) Compare patterns found in this study population with existing literature and/or accepted theories within the field. Hypotheses on changes in food patterns included: 1) Socio-economic status in the middle-class population maintained daily food production to remain inside the home; 2) Women working outside the home reduced labor hours allotted to home cooking; and 3) Social norms valued home cooking resulting in home prepared meals. Methods: The study used a cross-sectional, oral-history, interview format. The study sample consisted of 25 seniors $(65 y-100 y$ old $)$ from a convenience sample taken from one, medium-sized, mid-western town, Muncie, Indiana. The study involved use of a semi-structured, questionnaire/interview script, (approved by Ball State University's IRB committee). Results: Economics greatly influenced, and continues to influence, food consumption patterns for depression-era born adults. Women who grew up on home-only cooked meals, but entered the workforce adjusted traditional meals in favor of convenience. Implications: Health care providers trying to change dietary habits of older residents residing in the Midwest will need to consider foods and food preparatory methods introduced in childhood; these remained key components of the diet later in life and removing them may be met with resistance.
\end{abstract}

Keywords: Consumer Choice, Food Preference, Nutrition, Older Americans, Midwest

\section{Introduction}

Multiple times throughout the day people are making the decision of what to eat for their next meal. This seemingly easy decision is based upon numerous influences and life events. The elderly, markedly, will experience more influences and life course events as they have lived a longer time. Impacts on food choice are strongly influenced by life course events commencing with parental influence, then coming of age and early adult events, such as dating and marriage and establishing careers, followed by aging with the potential for new health diagnoses, and eventually the passing of a partner. All of these events are interlaced with other influences such as gender, income, and education level. These events and influences combine everyday to help one decide their food choices.

Parents are the initial influence for most people in making food choice decisions. Children will model parents' food choice behaviors at meals and snacks and are the principal source of information about food choices for the [1-4]. Dinner is the most common meal for families and where learned skills and taste preferences most commonly occur [5]. Research findings continually demonstrate that this parent is usually the mother, and people will set life-long standards for food preparation and taste based on their mother's preferences. Early impressions are so significant that they will persist through the life span with minor ebbs and flows occurring during life course events [6-8]. Though these initial impressions occur very early in life, they appear to set the standard for food as well as a source of comfort to turn to as one ages and experiences life events $[9,10]$. As people grow into adolescents, their food choices are highly influenced by peers in the short term by as much as $40 \%$ [11], but these influences have minimal influence on long term food choice ideals $[2,12,13]$. Although early childhood and adolescence is an abbreviated time span in a life, the impact of these initial influences and memories set the precedence for taste and food choice standards for the rest of an individual's life. 
In early adulthood, people expand in their social experience and potentially gain a spouse/partner during these years. Eating is a social ritual that helps create, enforce, and preserve relationships, whether friendship or with a romantic partner [14]. People will temporarily change eating patterns as they eat around different people in social settings. As people start romantic relationships they will even tailor food choices to attract budding partners, male or female [15-17]. As romantic relationships progress, multiple preferences and standards are attempting to merge, inclusive of point of view on how partners were raised as best practice [18]. As couples eat meals together, there is much consideration for the partner's preferred food choices. It was noted that women, more than men, were willing to be more flexible in food choices and compromise on their food choices to ensure pleasing meals between both partners $[19,20]$.

Income and education are determinates to food choice. As people expand their careers and combine incomes, they potentially should have secured more finances to have greater food purchasing power and increase foods purchased from all food categories. Men's education has been found to be more of an influence on food choices than women's education. This could imply that while household shoppers are mainly women, the products bought are highly influenced by the males' partiality [21].

Disease, physical and physiological changes can compound making people feel helpless and hopeless about the lack of control over food choices while depression can take over altering food choices [22]. As moods become increasingly negative, energy density of food increases resulting in weight gain $[23,24]$. Disease and the emotional changes that come with decreased health can alter food choices; this is prominent in the elderly.

Losing a partner can also influence food choices as people progress in age. When a partner passes, the other partner could experience changes in income, cooking style, and social surrounding and/or networking, all prospectively affecting food choices. Most elderly are on a fixed income and when one spouse is lost, finances can become even more restricted. Limited access to money severely modifies the ability to make desired food choices. Women are reported to be at a higher risk for food insufficiency and therefore are more likely to alter food choices away from preferred foods [25].

Elderly people consider having superior food skills an asset because they could still put together enjoyable meals with limited amounts of resources [1,26]. Though more elderly people are relying on dining out in restaurants because of convenience [27], the elderly would still prefer their meals be made from fresh ingredients over going out to eat [28]. This also allows them to control what is in their food better to help avoid foods that could cause any discomfort.

Occasionally the partner who was the primary food preparer will pass, forcing the other partner to either learn more skills or have the consequence of decreased food choice and potentially decreased nutritional health [29]. Widowed or single elderly people were more likely to opt for frozen or convenience foods than use or learn cooking skills $[28,30]$. When older adults ate with others, their eating was improved by $60 \%$ [31]. This study highlights the importance of older adults having social contact not only for a social connection but to also influence food choices positively.

Many determinants affect food choice, but consistently eight broad categories shape elderly food intake patterns. Research consistently shows that health status, convenience, life course, ideals, social norms, income, technology and product availability interact and change elderly food intake. The goal of this study was to measure the effect of these determinants, and to investigate geographical location as a modifier to the outcomes.

\section{Subjects and Methods}

\subsection{Methods}

The study used a cross-sectional, oral-history, interview format. The study sample consisted of 25 seniors $(65 \mathrm{y}-$ $100 \mathrm{y}$ old) from a convenience sample taken from one, medium-sized, mid-western town, Muncie, Indiana. The site was chosen to complement the 80 years of longitudinal demographic data collection commonly known as the Middletown Studies.

A semi-structured, questionnaire/interview script was developed and Ball State University's IRB committee granted approval. Participants were asked to recall what foods/meals they consumed while living with a parent, after they transitioned to be the primary food procurer/processor, and after leaving work (if applicable) and/or older than $65 \mathrm{y}$. Purposeful sampling was used to recruit participants of both genders and of varying ages, educational levels, income levels and living situations. Selection criteria was restricted to participants who were born and lived all but 8 years in Muncie, IN, excluding when the participant attended college and/or participate in the military.

Qualitative methods were used to uncover factors involved in the food choices made by a sample of older individuals. Interviews took place either in participants' homes, or the Cornerstone Center for the Arts, Community Center for Vital Aging all in the greater Muncie, Indiana geographic area. Informed consent to participate was collected and participants could remain in the study even if they chose not to grant permission for the inter- 
views to be uploaded into the Middletown Center data base. Interviews ranged between 50 and 75 minutes to complete, and were audio taped. The auto transcripts were transcribed verbatim, and entered into an excel worksheet and line numbered. Data analysis using the constant comparative method occurred throughout the data collection process [32]. This method of analysis involved coding each interview transcript initially for general concepts that emerged from the interviews. A tally of concepts was performed with each interview to indicate the degree of meaningfulness to the participant. The more times a concept appeared in the coding, the more this was noted to compare against all transcripts. Salient themes common to all transcripts were listed as emerging categories. Categories from all transcripts were entered into a spreadsheet to organize the data. Each participant's interview was compared to the categories to determine weighted significance.

Field notes were taken during and immediately after the interviews to identify question structure that seemed to build and/or eliminate barriers to engagement. Subsequent interviews were then reformatted to support an environment that created a safer interaction for the participant to share memories [33]. Successful interviews where dialog flowed effortlessly were replicated in the remaining interviews.

\subsection{Results}

Factors shaping the food choices of older study participants are presented in Figure 1. Factors can be separated into two broad indicators: external locus of control (economics, market availability, technology, social norms) and internal (convenience, health status, ideals, life course).

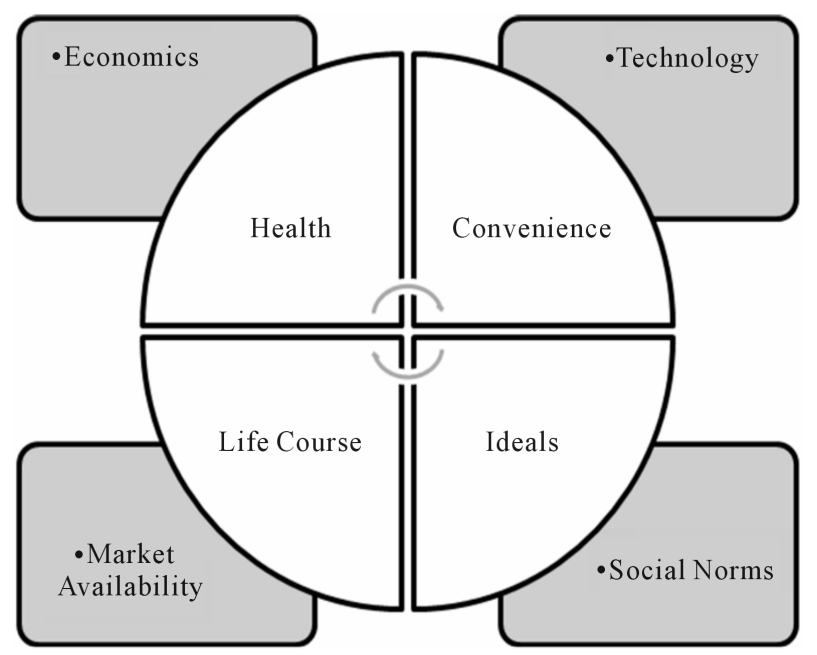

Figure 1. Influences on food choice among older adults living in a medium-sized, Midwest town.

\subsection{Economics}

All participants referenced monetary considerations as a factor to consumption. Each interview began with the same question soliciting the participant's earliest food memory. One female participant, age $78 \mathrm{y}$, began her interview with the following statement: I guess you could say I'm a depression baby. And continued with, so my early thoughts about food was [sic] more of food preparation. My parents um grew much as they could... canned as much as they could. So when we went to the grocery store it wasn't buying a lot of canned goods. Decades later frugality still played a significant role in many participants' lives. Another participant referenced that spending eight dollars on one meal in a restaurant was wasteful when you could go to the grocery store and purchase three to four days of groceries for the same amount of money. A female remarked: I can go to the grocery store and buy some flour and sugar, (laugh) I could do a whole lot with it. And I think that mentality is still there, spending all that money for one meal. Preparation of the meal was secondary to monetary costs.

Only one participant recalled dining in a restaurant before the age of twenty-one, and that event took place as part of travel away from home on a family vacation. Dining out in restaurants did not occur in childhood among the participants, however, later in life dining in family restaurants occurred on occasion as part of social events with friends or travel. Common answers among participants to dining-out included this statement from a 100 yr-old woman: No, we never went out and ate. Not when I was a kid. Never. Even after a long time we didn't go out to eat. Not like they do now that's for sure.

\subsection{Market Availability}

Twenty-five percent of participants mentioned they did not consume broccoli or cauliflower as children. An eight-six year-old, female remarked, I don't remember broccoli. We had potatoes, onions, carrots, beets. We had a lot of beets. Uh but we didn't have things like cauliflower or broccoli or asparagus. Foodstuffs that were locally produced in the geographic area compromised the diets of the participants until the 1970s when out of area items became available. After the pattern was detected (lack of broccoli and cauliflower consumption) the principal investigator $[\mathrm{PI}]$ queried the remaining participants on these two foodstuffs. None of the participants could recall when broccoli was introduced into the supermarkets, but many remarked that they had adopted the product into their current diets. Fish was another item that was not consumed until later in life. Snack foods were limited to home produced baked goods with the exception of one participant whose father received candy as a 
business gift and gave the products to his children. All participants commented that fruit was part of their daily lives growing up.

\section{Social Norms}

All participants were raised in households where the mother was primarily responsible for food production in the kitchen. All participants, while living with their parents, experienced vegetable gardens in their backyards or a community garden, where vegetables were grown. It was expected that the mother produce the meals, except in one household where the father was killed while working and the mother needed to work to support the three children. In this household the community and church supplemented meals to the family.

Children were expected to assist in various food-production, related chores, such as weeding the garden or washing the dishes, but none of the participants held a significant position in creating the daily meals. When it came to larger food production tasks, such as canning, most participants were expected to assist their parents. In the late 1920s and through the 1930s canning was a community event. The oldest (100 y) female participant commented as a young wife: Went up to Ball Center, they had a canning place on Macedonia [Ave], I think. I had to wash them and put them in the cans to get them ready and then they cooked them. Under pressure cooker I imagine. And when they got cool I brought them home. This participant during this time milked her own cow for milk, butter and cheese and raised pigs where the meat was canned for use in the winter. There were eight children in the household.

The older $(>80 \mathrm{y})$ participants experienced the greatest financial impact of the Great Depression. Men were unemployed and food availability, if not home produced was scarce. A male, African-American, $82 \mathrm{y}$, explained: around dinner time, supper three or four o clock in the afternoon there would be some women there in the church and you can go there and eat. Might just be ham and beans and corn and bread or something but it was food that you could eat and survive on. Follow-up questions revealed that this shortage of food influenced consumption patterns later in life. Distain for 21 st century youth's "food selectivity" is echoed in this male $(87 \mathrm{y})$ participant's remarks regarding his grandchildren: my mother put the food on the table and that's what we ate. And you pass it around and that's all we had and I never heard I'll fix you this if you don't like it. He continued that he felt this normative process taught him to accept military food while some of his fellow soldiers had a hard time adjusting to the taste of the food.

Participants all remarked that the dinner meal was a time when the family gathered around a table and shared a meal while growing up. Family was defined differently for some participants. Four participants had fathers that worked over the dinner hour. Parents' work schedules were accommodated. A participant's father was a firefighter, and he explained: we either had an early dinner in the evenings or a late dinner depending on which day he was scheduled for. Somewhere about 4:30 we would eat and he would leave for the fire department... It is significant that this family dined together, even though the father did not eat; rather, he ate dinner with his firemen colleagues later in the evening. When queried, participants remarked that they participated in school related sports, music and other extracurricular events, but the activities were scheduled prior to, or after, the $5-6 \mathrm{pm}$ dinner hour. The family dinner hour experienced as children was not maintained when the participants raised their own families, however.

Seventy-eight percent of the female participants worked outside the home while raising children. The females remarked how working outside the home shifted eating as a family unit and food preparation. A woman, a nurse, in her early 70 s who worked the evening shift felt guilty about not teaching her children to cook: none of my children learn to cook because I didn't take the time to teach them. And to this day they don't like to cook. It's not very nice. I have to look at myself in the mirror for that one. You can always just fight it and say I had to work. Yea, but there's occasions were you could teach them even a simple breakfast. Get them in there, but you know I didn't want them to mess up the kitchen. During the late 50 s and into the early 60 s technology impacted food production and women incorporated timesaving food preparation conveniences..

\section{Technology}

Another nurse who grew up on a farm during the depression and placed cost as her deciding determinant to food consumption, relented and purchased prepared goods when the food cost was low enough to negate financial concerns. She recalled: My parents didn't have the money...now if I got four dollars and fifty-cents, why go buy (apples and sugar) when I got $\$ 4.98$ to go buy the jelly? Smucker's makes pretty good jelly. Having the financial resources and market availability given technological advances took precedence in food consumption decision making. The labor involved making jelly, even with extensive jelly making history, for this working female was not worth it. Tellingly, poorer sensory pleasure was accepted. I've gotten accustomed to it. So yes I'll eat it now, but no it does not taste like it used to. She continues, buy that (butterscotch) pie, it's good because you've come accustomed to eating it. It does not taste like that homemade pie. She reasons: the time factor is so signifi- 
cant that you are willing to have a little bit less tasteful of a product if you don't have to spend two hours making it. Another participant who consumed only home prepared foods as a child adopted frozen dinners later in life when they became available. She remarked: I use a lot of TV trays. I like Lean Cuisine and I like uh Stouffer's. This participant is the rare exception to consuming frozen prepared meals. Most have incorporated some snack foods rather than whole meals. Typical replacements were baked goods such as cakes and cookies

\section{Life Course}

As the participants aged eating patterns changed. Major events such as the introduction of children into the household, marriage, divorce, working outside the home and retirement shifted eating patterns. Childhood experiences shaped current food choices, but technology, market availability, social norms, economy all shaped the participants' life course as well. Men and women differed in change in eating patterns. Men tended to maintain the same diet throughout their lives. Women though were more likely to dictate food choices from the store and therefore also dictate food choices for meals because they "control" what comes into the house $[34,35]$. So even though women are more willing to compromise on meals eaten, they will determine ingredients and specific brands into each meal, creating a way to add their own standards within the meal. Because there is a compromise in cooking and attempt to please many palates at the table, people are likely to eat a diet that is more varied [36].

Women, upon marrying, learned their husbands eating habits and prepared meals to accommodate their preferences even at their own expense. A retired woman remarked: "Bill" doesn't like vegetables, very few vegetables he likes and that's always hard. I could, I could live off fruits and vegetables but I don't have them too often. We always have some vegetable always with our meal but I guess I just don't bother to fix them. You know.

When roles changed through divorce, eating patterns changed in tandem. A few participants remarked on the role divorce had on food intake. One woman raised her son as a single mom in the 1950s and conceded that up until the divorce she cooked all the meals, but when she returned to work she began purchasing convenience foods to shorten the food preparation time. It was the childhood experiences such as family dining together at dinner that became the barometer for acceptable or preferable eating behaviors even after 60 years.

\section{Ideals}

The two life shaping ideals among the participants included eating together as a family and accepting, without compliant, what was served to you. Food scarcity during the depression forever imprinted the significance of appreciating food availability. One 82 y-old male, participant remarked that during the depression: I remember a lot of time we didn't have anything to eat and they had something at the church and you could go to church and get you know a bowl of chili or some kind of foods, food whatever they had to eat. He continues speaking about hunting for and eating squirrel: yeah, I like to hunt when I got older, but it was a whole different... it wasn't a survival type of thing you know, when I was a kid, it was survival. Many of the participants expressed disgust at today's generation of children where parents cater to their likes and dislikes. A male 81y old participant commented: you ate what was on the table you know.

Although many participants evolved from sitting down to daily family meals when they were children, later in their adulthood those that had children in activities that ran over the $5-7 \mathrm{pm}$ dinner hour mentioned its significance. A male remarked: all three of my daughters were involved in sports...but we'd always eat as a family, ninety-nine percent of the time. There would be times when we would have to get something to eat when we got back late, but we did. On days where all members were free, and always on Sundays, families ate together as a unit.

Most participants, and all men, consumed meat and potatoes at every meal when they had the financial resources to do so. A male remarked: I can't remember ever having a meal without having some kind of meat on the table. Root vegetables such as carrots, turnips, beets were incorporated into the meals, but some men did not consume vegetables later in life except at their spouse's insistence. What constituted meals included meat, starch and vegetable. Vegetarian items were rare, but legumes such as navy beans and ham were commonplace.

\section{Health}

As couple's age, changes in health shift instituted food choices that had been established early in the relationship. Food choices are decided upon mostly based on familiarity and comfort [37,38], but with aging, ease of eating and health problems also change the way people eat. The aging process can naturally cause digestive difficulties that changes food tolerances, compelling people to change diets to accommodate new needs and avoid gastric dilemmas. Loss of dentition, weakening physical strength, and arthritis are additional ailments that potentially could make one adapt their diet to changing needs [6]. The ability to pick up and adequately use dining utensils and then properly chew food can decrease with these types of physical changes in older adults.

None of the participants remembered consuming food- 
stuffs for health-related reasons as children. As adults and with the influence of the media and doctors, some participants changed their diets for health related concerns. The predominant change included switching from butter to margarine to help lower cholesterol. A 78 y-old male thought that he was a rare "in-the-know" person about the relationship between butter and cholesterol: you know, you start using your own thoughts as to how you can bring your cholesterol down...it would be interesting to know what the cholesterol [of] some of the older people in my growing up years was because they had, they didn't have a clue what a cholesterol was all about. And they did not use anything... I mean they used everything that had a high cholesterol count. But they survived. We all grew up and our parents wasn't (sic.) concerned with it. The fact that their peers consumed a high cholesterol diet for over $60 \mathrm{y}$ and are still healthy in their 80 s seemed to contradict what they read and heard from their doctors. However, this population holds doctors' medical expertise in high esteem and therefore they changed their diets to margarine, even though they preferred the taste of butter. Women were more likely to adhere to a higher fruit, vegetable, lower animal product diet than men, and collect health information from popular lay print material.

One male commented how his missed the old days when calories and fat were not a consideration. Regarding his wife, the gatekeeper to meals, he remarked: "Linda" didn't like it too well but I fixed a skillet with a few pieces of chicken in lard and she didn't know but if I could go back and have Mom fix a great big old thing of fried chicken, mashed potatoes and that gravy from all the stuff left in the skillet. Well I did that.

\section{Convenience}

During the 1920s and 30s takeout food was mostly unavailable. One participant whose father was a salesman who sold paper products to a few local restaurants claimed to invent the Chinese takeout container. She offered: my dad did love Chinese food and he would go down to Jackson Street and park in the Alley behind this restaurant. We never went in there and sat down to be waited on but he'd go down the alley and go in the back way... this was probably in the early 40's and it was during the second World War ... my dad would tease with the head cook and he would put in Chop Suey... and he would put in these white buckets and fold it over. Just like the Chinese takeout today. When takeout food became available participants remarked that the cost was prohibitive until after the children were schooled or married and then only on occasion.

Foods common to takeout include ethnic type foods such as Chinese, Thai, pizza or Indian. Both men and women commented that they do not care for ethnic cuisine aside from the occasional pizza. One woman remarked that she did not like pizza, but while in college in the 50s she was consumed pizza to fit in with her peers. Restaurant patronage entered the majority of the participants' lives as retirees. Even then, cost is still the determining factor with dining out with breakfast the most common meal consumed out of the house. Frozen meat items such as fried chicken or Salisbury steaks purchased from supermarkets are rarely consumed. However, frozen vegetables are widely incorporated into meals and appreciated for the reduction in meal preparation time.

\section{Discussion}

This research sought to delineate an understanding of food choice processes among older adults living in a medium-sized Midwestern town while proposing a theoretical model specific to this age group. Prior research with this older population had been primarily conducted within states that border the United States coasts. Life in the semi-rural Midwest prior to, during, and shortly after the Great Depression differently affected this population. Most families were linked to farming with all but a select few growing vegetables on their properties. During the 1920 s through the 1930s families were self-sufficientraised farm animals, commodities and fruits and vegetables. Many of the participants remarked that food shortages during WWII did not greatly affect them. Fruit was plentiful and summer harvests were canned for winter use.

Lack of income during the Depression defined ideals, including food consumption forevermore. Food was, and continues to be, valued as a gift not to be taken for granted. Selectivity is frowned upon. Dinner meals brought the family together and when participants reached maturity they tried to incorporate some semblance of earlier childhood dining experiences, even if only on Sundays. Community meals held at the church brought families across town together and continue to draw participants later in life to communal dining. All but one individual who just recently entered an assisted living home prepared home cooked meals. The oldest (female 100 y old) participant's son who lives next door cooks for his mother.

Managing two careers and potentially children, creates time constraints and difficulties in planning meals for families to eat together. Employment itself can influence food choices because of two people having to work around different work schedules and other demands from work [39]. Work schedules can aid in food choice by establishing time frames for when families or couples eat, foods that would be convenient for eating at that time, and environmental conditions that will surround the dinner. It also assists in developing the routine in which the family and/or couple eat if the work schedules are similar 
week to week [40]. Meals and specific food choices in families are largely determined by convenience and time factors, such as ease of preparation and work schedules, respectively [20] and this was demonstrated in this study. Demanding schedules combined with the need for quality time with family members make dinner time a priority for creating a pleasing meal for all a priority. Dinner, for most, will be the only meal eaten together during the day, and partners are attempting to create a positive environment during this limited time together [41]. Seventy-five percent of the participants mentioned the change in attitude that their children held towards skipping the dinner meal so that they could assist their children in particiapting in sporting events.

Physiological changes in taste also occur as a natural aging process $[25,42]$. As people age, there is approximately a one percent loss in olfactory nerve fibers that greatly affect taste and therefore food choice [43]. When odors are not detected by olfactory nerves, taste is greatly affected and preferred foods stop becoming food choices, because favored foods taste unappetizing or different from their standard established in childhood. Losing olfactory senses over time can decrease the ability to correctly choose foods that are not decaying and decrease positive associations with food choice and odors [42]. Physical and physiological changes that occur during the aging process can vastly effect food choices made either because of ability eat foods, mechanically and gastric tolerance, or because they create changes in taste that are unappealing to the palate. Taste preference played a lesser role than convenience and economics in this population. Even with detectable taste differences, participants' choices were more determined by other factors than taste.

Diabetes and heart disease are prominent diseases that also trigger major changes to food choice. While disease will affect most people, women are more likely to pattern food choices based on health then men overall [44]. This trend though was discovered to disappear as people aged [45], where most elderly males and females were inspired to eat healthier. Participants in this study at the higher end of the age spectrum did alter food consumption to follow advice given by their doctors. Food choices changed specifically by the lowering of fat and cholesterol intake, and making overall healthier food choices. The health status of the participants and advice received greatly affected food choice. Healthcare professionals advising this population need to align nutrition messages with doctor recommendations. Our outcomes are supported by recent research findings [30]. Disease states might alter the way people eat urging them to avoid favorite foods, but people appear more willing to modify foods if it will allow them to live longer and help avoid further advancement in their disease or new diseases in the future.

\section{Conclusions}

This study added new insights into food consumption patterns among older Americans. While the majority of the findings are congruent with studies on the elderly some differences were found. Unlike other findings, taste played a secondary role to food consumption especially among women. This may be a regional issue where frugality and farming self sufficiency is highly valued. Monetary considerations and convenience were predictors to food consumption with taste compromised if ease of preparation was strengthened. Existing literature suggests that taste is the primary determinant to food consumption. While most participants waned over childhood eating patterns that evoked taste memories, favorite foods among them butter were drastically reduced or eliminated.

Eating patterns were separated into two broad categories where internal and external forces shaped food choice. These choices were not stagnant; rather, they evolved along with the participant's life course. Marriage, childbirth, divorce, retirement and health status constantly were in flux coupled with changing food technology and shifting social norms, all these variables moved forward or receded singularly or grouped and determined food choice.

Health care providers designing programs to influence food consumption will benefit from this research. Meal preparation advice based on low-cost, extreme ease in preparation (including cleanup), meat and potato ingredients will have a greater acceptance with this population. Strategies on dining out with healthier choices with an emphasis on breakfast items may be well received. Convincing this male population to consume increased vegetables may be counterproductive and shut down avenues of communication. Doctors are a respected group for diet advice and providing materials for their patients while sitting in the waiting room is an opportunity to explore.

When interpreting the results of this study, limitations should be recognized. All participants were self-selected and received a fifteen dollar gift card to the local grocer. Social economic level and gender was stratified; however, the education level was skewed toward post secondary education, with $68 \%$ of the population completing at least some college. As qualitative research, this study relied on self-reports of the participants. Participants were free to share food memories, but may have held back what is considered undesirable by society. Findings may not necessarily be generalized to groups outside this geographic region.

The significance of these variables in determining food consumption patterns and its roles in lifelong behaviors 
patterns have important implications for nutrition education, research, and practice. Many food choices are developed in early childhood and recording a person's life progression illuminates avenues for intervention and understanding. Further retrospective and longitudinal studies could support current findings and take the research to a deeper level.

\section{Acknowledgements}

The authors thank the participants in this study for their time and effort, and Judy Elton for participant recruitment. Funding for this study came in part from a Ball State University Faculty ASPiRE grant. No conflicts of interest are reported.

\section{REFERENCES}

[1] C. A. Bisogni, M. Jastran, L. Shen and C. M. Devine, "Biographical Study of Food Choice Capacity: Standards, Circumstances, and Food Management Skills," Journal of Nutrition Education and Behavior, Vol. 37, No. 6, 2006, pp. 284-291. doi:10.1016/S1499-4046(06)60158-9

[2] M. M. Bissonnette and I. R. Contento, "Adolescents' Perspectives and Food Choice Behaviors in Terms of the Environmental Impacts of Food Production Practices: Application of a Psychosocial Model," Journal of Nutrition Education, Vol. 33, No. 2, 2001, pp. 72-82. doi:10.1016/S1499-4046(06)60170-X

[3] R. Brown and J. Ogden, "Children's Eating Attitudes and Behavior: A Study of the Modeling and Control Theories of Parental Influence," Health Education Research, Vol. 19, No. 3, 2004, pp. 261-271.doi:10.1093/her/cyg040

[4] J. O. Fisher and L. L. Birch, "Restricting Access to Foods and Children's Eating," Appetite, Vol. 32, No. 3, 1999, pp. 405-419. doi:10.1006/appe.1999.0231

[5] C. E. Blake, C. A. Bisogni, J. Sobal, M. Jastran and C. M. Devine, "How Adults Construct Evening Meals. Scripts for Food Choice," Appetite, Vol. 51. No. 3, 2008, pp. 654-662. doi:10.1016/j.appet.2008.05.062

[6] L. W. Falk, C. A. Bisogni and J. Sobal, "Food Choice Process of Older Adults: A Qualitative Investigation," Journal of Nutrition Education, Vol. 28, No. 5, 1996, pp. 257-265. doi:10.1016/S0022-3182(96)70098-5

[7] C. M. Devine, M. Connors, C. A. Bisogni and J. Sobal, "Life-Course Influences on Fruit and Vegetable Trajectories: Qualitative Analysis of Food Choices," Journal of Nutrition Education, Vol. 30, No. 6, 1998, pp. 361-370. doi:10.1016/S0022-3182(98)70358-9

[8] C. M. Devine, "A Life Course Perspective: Understanding Food Choices in Time, Social Location, and History," Journal of Nutrition Education and Behavior, Vol. 37, No. 3, 2005, pp. 121-128. doi:10.1016/S1499-4046(06)60266-2

[9] P. A. Hannon, D. J. Bowen, C. M. Moinpour and D. F. McLerran, "Correlations in Perceived Food Use Between the Family Food Preparer and Their Spouses and Chil- dren," Appetite, Vol. 40, No. 1, 2003. pp. 77-83. doi:10.1016/S0195-6663(02)00140-X

[10] I. R. Contento, S. S. Williams, J. L. Michela and A. B. Franklin, "Understanding the Food Choice Process of Adolescents in the Context of Family and Friends," Journal of Adolescent Health, Vol. 38, No. 5, 2006, pp. 575582. doi:10.1016/j.jadohealth.2005.05.025

[11] J. M. Decastro, "Family and Friends Produce Greater Social Facilitation of Food Intake than Other Companions," Physiology and Behavior, Vol. 56, No. 3, 1994, pp. 445-455. doi:10.1016/0031-9384(94)90286-0

[12] T. Furst, M. Connors, C. A. Bisogni, J. Sobal and L. W. Falk, "Food Choice: A Conceptual Model of the Process," Appetite, Vol. 26, No. 3, 1996, pp. 247-265. doi:10.1006/appe.1996.0019

[13] I. De Bourdeaudhuij and J. Sallis, "Relative Contribution of Psychosocial Variables to the Explanation of Physical Activity in Three Population-Based Adult Samples," Preventive Medicine, Vol. 34, No. 2, 2002, pp. 279-288. doi:10.1006/pmed.2001.0979

[14] J. Sobal and, M. K. Nelson, "Commensal Eating Patterns: A Community Study," Appetite, Vol. 41, No. 2, 2003, pp. 181-190. doi:10.1016/S0195-6663(03)00078-3

[15] D. E. Amiraian and J. Sobal, "Dating and Eating. Beliefs about Dating Foods Among University Students," Appetite, Vol. 53, No. 2, 2009, pp. 226-232. doi:10.1016/j.appet.2009.06.012

[16] S. J. Salvy, L. R. Vartanian, J. S. Coelho, D. Jarrin and P. P. Pliner, "The Role of Familiarity on Modeling of Eating and Food Consumption in Children," Appetite, Vol. 50, No. 2, 2008, pp. 514-518. doi:10.1016/j.appet.2007.10.009

[17] M. E. Young, M. Mizzau, N. T. Mai, A. Sirisegaram and M. Wilson, "Food For Thought. What You Eat Depends on Your Sex and Eating Companions," Appetite, Vol. 53, No. 2, 2009, pp. 268-271. doi:10.1016/j.appet.2009.07.021

[18] C. A. Bisogni, M. Connors, C. M. Devine and J. Sobal, "Who We Are and How We Eat: A Qualitative Study of Identities in Food Choice," Journal of Nutrition Education and Behavior, Vol. 34, No. 3, 2002, pp. 128-139. doi:10.1016/S1499-4046(06)60082-1

[19] M. Connors, C. A. Bisogni, J. Sobal and C. M. Devine, "Managing Values in Personal Food Systems," Appetite, Vol. 36. No. 3, 2001, pp. 189-200. doi:10.1006/appe.2001.0400

[20] C. F. Bove, J. Sobal and B. S. Rauschenbach, "Food Choices Among Newly Married Couples: Convergence, Conflict, Individualism, and Projects," Appetite, Vol. 40, No. 1, 2003, pp. 25-41. doi:10.1016/S0195-6663(02)00147-2

[21] L. Ricciuto, V. Tarasuk and A. Yatchew, Socio- demographic Influences on Food Purchasing Among Canadian Households," European Journal of Clinical Nutrition, Vol. 60, No. 6, 2006, pp. 778-790. doi: $10.1038 /$ sj.ejen. 1602382

[22] M. A. Fiatarone, E. F. O'Neill, N. D. Ryan, K. M. Cle- 
ments, G. R. Solares and M. E. Nelson, "Exercise Training and Nutritional Supplementation for Physical Frailty in Very Elderly People," New England Journal of Medicine, Vol. 330, No. 25, 1994, pp. 1769-1775. doi:10.1056/NEJM199406233302501

[23] E. L. Gibson, "Emotional Influences on Food Choice: Sensory, Physiological and Psychological Pathways," Physiology and Behavior, Vol. 89, No. 1, 2006, pp. 53-61. doi:10.1016/i.physbeh.2006.01.024

[24] R. W. Jeffery, J. A. Linde, G. E. Simon, E. J. Ludman, P. Rohde and L. E. Ichikawa, "Reported Food Choices in Older Women in Relation to Body Mass Index and Depressive Symptom," Appetite, Vol. 52, No. 1, 2009, pp. 238-240. doi:10.1016/j.appet.2008.08.008

[25] J. R. Sharkey, "Diet and Health Outcomes in Vulnerable Populations," Annals of The New York Academy of Sciences, Vol. 1136, No. 1, 2008, pp. 210-217. doi:10.1196/annals.1425.020

[26] M. P. Lawton and E. M. Brody, "Assessment of Older People: Self-maintaining and Instrumental Activities of Daily Living," Gerontologist, Vol. 9, No. 3, 1969, pp. 179-186. doi:10.1093/geront/9.3 Part 1.179

[27] A. K. Kant and B. I. Graubard, "Eating Out in America, 1987-2000: Trends and Nutritional Correlates," Preventive Medicine, Vol. 38, No. 2, 2004, pp. 243-249. doi:10.1016/j.ypmed.2003.10.004

[28] W. Hunter and T. Worsley, "Understanding the Older Food Consumer. Present Day Behaviors and Future Expectations," Appetite, Vol. 52, No. 1, 2009, pp. 147-154. doi:10.1016/j.appet.2008.09.007

[29] S. A. Quandt, J. McDonald, T. A. Arcury, R. Bella and M. Z. Vitolins, "Nutritional Self-management of Elderly Widows in Rural Communities," Gerontologist, Vol. 40, No. 1, 2000, pp. 86-96. doi:10.1093/geront/40.1.86

[30] G. Hughes, K. M. Bennett and M. A. Hetherington, "Old and Alone: Barriers to Healthy Eating in Older Men Living on Their Own," Appetite, Vol. 43, No. 3, 2004, pp. 269-276. doi:10.1016/j.appet.2004.06.002

[31] S. J. McAlpine, J. Harper, M. T., McMurd, C. BoltonSmith and M. M. Hetherington, "Nutritional Supplementation in Older Adults: Pleasantness, Preference and Selection of Sip-feeds," British Journal of Health Psychology, Vol. 8, No. 1, 2003, pp. 57-66. doi:10.1348/135910703762879200

[32] E. Glaser and A. L. Strauss "Discovery Grounded Theory: Strategies for Qualitative Research,” Aldine, Chicago, 1967.

[33] H. Miles and M. Huberman "Qualitative Data Analysis: A sourcebook of new methods," Aldine, Chicago, 1984.

[34] R. Bassett, G. E. Chapman and B. L. Beagan, "Autonomy and Control: The Co-Construction of Adolescent Food Choice," Appetite, Vol. 50 No. 2, 2008, pp. 325-332. doi:10.1016/j.appet.2007.08.009

[35] N. Sudo, D. Degeneffe, H. Vue, K. Ghosh and M. Reicks,
"Relationship Between Needs Driving Eating Occasions and Eating Behavior in Midlife Women," Appetite, Vol. 52, No. 1, 2009, pp. 137-146. doi:10.1016/j.appet.2008.09.005

[36] V. B. Duffy, J. R. Backstrand and A. M. Ferris, "Olfactory Dysfuction and Related Nutritional Risk in Free-Living, Elderly Women," Journal of the American Dietetic Association, Vol. 95, No. 8, 1995, pp. 879-884. doi:10.1016/S0002-8223(95)00244-8

[37] A. Steptoe, T. M. Pollard and J. Wardle, "Development of a Measure of the Motives Underlying the Selection of Food: The Food Choice Questionnaire," Appetite, Vol. 25, No. 3, 1995, pp. 267-284. doi:10.1006/appe.1995.0061

[38] Z. Pieniak, W. Verbeke, F. Vanhonacker, L. Guerrero and M. Hersleth, "Association between Traditional Food Consumption and Motives for Food Choice in Six European Countries," Appetite, Vol. 53, No. 1, 2009, pp. 101-108. doi:10.1016/j.appet.2009.05.019

[39] A. A. Lake, A. J. Rugg-Gunn, R. M. Hyland, C. E. Wood, J. C. Mathers and A. J. Adamson, "Longitudinal Dietary Change from Adolescence to Adulthood: Perceptions, Attributions and Evidence," Appetite, Vol. 42, No. 3, 2004, pp. 255-263. doi:10.1016/j.appet.2003.11.008

[40] M. M. Jastran, C. A. Bisogni, J. Sobal, C. Blake and C. M. Devine, "Eating Routines. Embedded, Value Based, Modifiable, and Reflective," Appetite, Vol. 52, No.1, 2009, pp. 127-136. doi:10.1016/j.appet.2008.09.003

[41] C. M. Devine, M. Jastran, J. Jabs, E. Wethington, T. J. Farell and C. A. Bisogni, "A Lot of Sacrifices: WorkFamily Spillover and the Food Choice Coping Strategies of Low-Wage Employed Parents," Social Science and Medicine, Vol. 63, No. 10, 2006, pp. 2591-2603. doi:10.1016/j.socscimed.2006.06.029

[42] C. J. Wysocki and M. L. Pelchat, "The Effects of Aging on the Human Sense of Smell and its Relationship to Food Choice," Critical Reviews in Food Science and Nutrition, Vol. 33, No. 1, 1993, pp. 63-82. doi:10.1080/10408399309527613

[43] C. G. Smith, "Age Incidence of Atrophy of Olfactory Nerves in Man. A Contribution to the Study of the Process of Aging," The Journal of Comparative Neurology, Vol. 77. No. 3, 1942, pp. 589-595. doi:10.1002/cne.900770306

[44] M. Ree, N. Riediger and M. H. Moghadasian, "Factors Affecting Food Selection in Canadian Population," European Journal of Clinical Nutrition, Vol. 62, No. 11, 2008 , pp. 1255-1262. doi:10.1038/sj.ejen.1602863

[45] J. L. Locher, C. S. Ritchie, D. L. Roth, B. Sen, K. S. Vickers and L. I. Vailas, "Food Choice Among Homebound Older Adults: Motivations and Perceived Barriers," The Journal Of Nutrition, Health and Aging, Vol. 13, No. 8, 2009, pp. 659-664. doi:10.1007/s12603-009-0194-7 